\title{
Modeling the impact of temperature on fractional order dengue model with vertical transmission
}

\author{
Ozlem Defterli* $^{*}$ \\ Department of Mathematics, Faculty of Arts and Sciences, Cankaya University, 06790 Ankara, Turkey \\ defterli@cankaya.edu.tr
}

\begin{tabular}{|c|c|}
\hline ARTICLE INFO & ABSTRACT \\
\hline $\begin{array}{l}\text { Article History: } \\
\text { Received } 11 \text { September } 2019 \\
\text { Accepted } 23 \text { October } 2019 \\
\text { Available } 28 \text { January } 2020\end{array}$ & \multirow{3}{*}{$\begin{array}{l}\text { A dengue epidemic model with fractional order derivative is formulated to an- } \\
\text { alyze the effect of temperature on the spread of the vector-host transmitted } \\
\text { dengue disease. The model is composed of a system of fractional order differ- } \\
\text { ential equations formulated within Caputo fractional operator. The stability } \\
\text { of the equilibrium points of the considered dengue model is studied. The cor- } \\
\text { responding basic reproduction number } R_{0}^{\alpha} \text { is derived and it is proved that if } \\
R_{0}^{\alpha}<1 \text {, the disease-free equilibrium (DFE) is locally asymptotically stable. } L 1 \\
\text { method is applied to solve the dengue model numerically. Finally, numerical } \\
\text { simulations are also presented to illustrate the analytical results showing the } \\
\text { influence of the temperature on the dynamics of the vector-host interaction in } \\
\text { dengue epidemics. }\end{array}$} \\
\hline $\begin{array}{l}\text { Keywords: } \\
\text { Fractional operators } \\
\text { Stability of the equilibria } \\
\text { Dengue epidemics } \\
\text { Temperature effect }\end{array}$ & \\
\hline $\begin{array}{l}\text { AMS Classification 2010: } \\
\text { 26A33; 34A34; 92B05; 92D30 }\end{array}$ & \\
\hline
\end{tabular}

\section{Introduction}

A deeper understanding of mathematical models is essential to represent and to reliably control the transmission of the (epidemic/pandemic)diseases. The vector-borne diseases becomes an extensive threat with a significant affect on human and animal health. Distribution of vector-borne diseases is determined by complex demographic, environmental and social factors. Vector-borne diseases are responsible from more than 700000 deaths annually as taking a part of $17 \%$ of all infectious diseases. Dengue fever, as a severe, flu-like illness influences infants, young children and adults, is mainly faced in urban and semi-urban areas of the countries in tropical and sub-tropical climates [1,2]. Dengue fever disease has no concrete treatment but early detection and efficient medical treatment reduces the death rates below $1 \%$.

Dengue virus is carried from vector-host-vector mainly by the bites of infected female mosquitoes of the type Aedes aegypti. After virus incubation for 410 days, an infected mosquito is able to transmit the virus for the rest of its life [2].
All mosquito species go through four distinct phases during their life cycle: Egg, Larva (plural: larvae), Pupa (plural: pupae), Adult. The first three phases take place in water, but the adult is an active flying insect (see Figure 1). Only the female mosquitoes bite and they feed on the blood of humans or other animals.

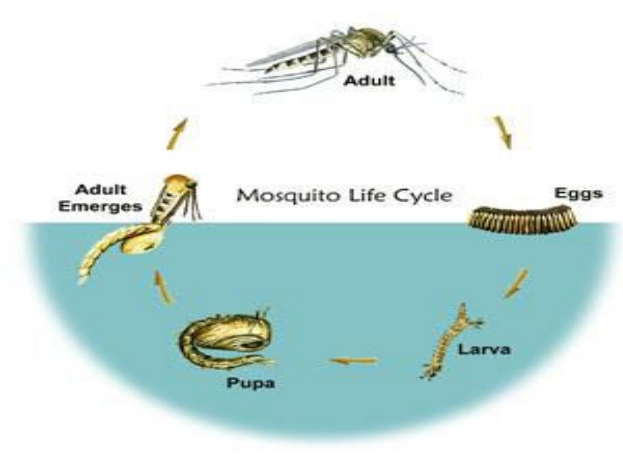

Figure 1. Mosquito Life Cycle 3].

In order to keep the dengue infection under control some adequate and powerful mathematical (compartmental) models and analysis have been 
suggested (see [4 15]). Abdelrazec et al. [15] developed a model for the dynamic study of transmission of dengue fever by means of a nonlinear rate of recovery to analyze the spread and control of the disease. A mathematical model for dengue is constructed in [8] to determine the chain of two epidemic diseases with different human populations. The reproduction number $R_{0}^{\alpha}$ as a threshold quantity of the epidemics is explained by means of the stability analysis. Their model shown that the ecological administration alone as a the vector control is not enough; it may postpone the spread of epidemics. The usage of a vaccine may control simultaneously against some serotypes. In Andraud et al. [16], deterministic models of dengue transmission are surveyed in terms of the assumptions for parameters, threshold values and control measures. The effect of seasonal variations in temperature and some other climate factors on the transmission dynamics of dengue diseases are epidemiologically discusses in several experimental research (e.g. see 17] the references therein) and mathematically analyzed in the recent studies [18 21]. The mathematical analysis in these references are based on the compartmental integer-order epidemic models that contain a system of ODE's. However, in general, integer-order systems are memoryless ( 4, 8, 10, 11, 15, 20]).

Fractional calculus is the study of an extended form of integrals and derivatives in fractional orders. The most vital aspect of fractional derivatives is that, the models based on these operators hold memory which provides an important advantage for a well understanding of the behaviour of the entomological factors and so the dynamics of the epidemic diseases. Sardar et al. [12 investigated a compartmental dengue transmission model having the memory, in which the memory incorporated in the model exhibiting an arbitrary order differential operator. A threshold quantity $R_{0}^{\alpha}$ is derived, having the similarity with elementary reproduction number and determined that although the value of $R_{0}^{\alpha}$ is less than one, the disease-free equilibrium would not be constantly stable, and the system reveals a Hopf-type bifurcation. The SIR model of the fractional order differential equation of the dengue fever is investigated in [22], and a fractional order SEIR model with vertical transmission in a nonconstant population is studied by [23]. In [24] and 25], three definitions of fractional operators are carried out for MSEIR models of some other type of epidemics as varicella disease validated by an outbreak data. Moreover, in the studies of [26 29], dengue fever epidemics is modeled within real data by the help of fractional operators of some types that varies by the definition of their kernel. Our goal in this study is to investigate the fractional order dynamical model of the dengue fever with temperature effect centered at the distribution of the human population into three categories (susceptible, infected, and resistant humans), whereas the population of the Aedes aegypti pre-adult female mosquitos (eggs and larvae) is distributed in two parts (susceptible and infected) and adult mosquitos population is divided in three parts (susceptible, infected but not infectious, and infectious) to understand the dynamics of dengue disease in a more accurate and realistic way.

The manuscript is organized as follows: after the related literature info in Introduction part in Section 1, the generalized non-integer order mathematical model of the temperature effect in the vector-host (vertical) transmission dynamics of dengue fever epidemics is introduced in Section 2 with a preliminary info about its parameter list and initial conditions. The disease-free equilibrium points of the system are newly obtained and the local asymptotic stability conditions are derived correspondingly which results the basic reproduction number of the system. Section 3 is dedicated to the numerical solution of the discretized version of generalized Caputo-based dengue model. Followingly, the numerical simulations are performed for the model (for different temperature values and fractional orders) in order to analyze the temperature effect on the dengue transmission dynamics by using the real data of the FongShan district, Kaohsiung, Taiwan. The discussions of the simulations are provided in Section 3 and the concluding remarks are given in Section 4.

\section{Preliminaries and the proposed generalized dengue model}

In this part, we present fractional operators where the Caputo fractional derivative is mainly considered. For a function $x(t)$ defined on a time interval $[0, T]$, the Caputo derivative and integral of $x(t)$ are denoted by ${ }^{C} D^{\alpha} x(t)$ and ${ }^{C} I^{\alpha} x(t)$, respectively, and defined as 30

$$
\begin{aligned}
{ }^{C} D^{\alpha} x(t) & :=\frac{1}{\Gamma(1-\alpha)} \int_{0}^{t}(t-\xi)^{-\alpha} \dot{x}(\xi) d \xi, \\
{ }^{C} I^{\alpha} x(t) & :=\frac{1}{\Gamma(\alpha)} \int_{0}^{t}(t-\xi)^{\alpha-1} x(\xi) d \xi,
\end{aligned}
$$

where $0<\alpha<1$ represents the order of the fractional operator. 
We will examine a mathematical model of the dengue fever having arbitrary order, the parameter values used in this mathematical model are estimated based on the demographic data of FongShan district, Kaohsiung in southern Taiwan ( [20], [31]). Kaohshiung is the second largest cosmopolitan city of Taiwan. The data showed that Kaohsiung was at high risk for dengue fever from 1998 to 2010 and in total 2415 number of cases of dengue fever were reported. According to the WHO Dengue situation update by July 2019, approx. 29300 cases reported in 2019 only in the Western Pasific Region ( [1], [2]). In this study, a set of weekly meteorological data has been used which belongs to Kaohsiung district that was recorded by 11 supervising observatory locations of the Taiwan Environmental Protection Agency 3 in 2011, further weekly maximum, minimum and mean temperatures between the years of 2001 and 2010 are incorporated 20].

\subsection{Fractional order vector-host dengue model}

This study is based upon the model of vectorhost transmission dynamics proposed in 32 to represent the transmission patterns of the dengue fever. The population has been separated into three main parts as host (human), vector (preadult female mosquito), and vector (adult female mosquito) population. There are two compartments of Aedes aegypti pre-adult female mosquito population (effective eggs and larvae) namely susceptible $E_{s}$ and infected $E_{i}$. The three compartments of vector (adult) population are stated accordingly as: $M_{s}, M_{e}$, and $M_{i}$, which are the values evaluated at time $t$ of susceptible, infected (but not being infectious) and infectious female mosquitoes. Also, host (human population) is divided into three compartments as: $H_{s}, H_{i}$, and $H_{r}$, these are the numbers at the time $t$ of the susceptible, infected/infectious and recovered/immune human populations, respectively. This way dengue model defined by the following system [32] and correspondingly the model parameters are listed in Table 1 .

$$
\left\{\begin{array}{l}
{ }^{C} D^{\alpha} E_{s}=e_{v}^{\alpha}\left(1-p\left(\frac{M_{i}}{M_{s}+M_{e}+M_{i}}\right)\right)-\eta^{\alpha} E_{s}, \\
{ }^{C} D^{\alpha} E_{i}=e_{v}^{\alpha} p\left(\frac{M_{i}}{M_{s}+M_{e}+M_{i}}\right)-\eta^{\alpha} E_{i}, \\
{ }^{C} D^{\alpha} M_{s}=\eta^{\alpha} E_{s}-b^{\alpha} \frac{H_{i}}{N_{h}} M_{s}-\delta^{\alpha} M_{s}, \\
{ }^{C} D^{\alpha} M_{e}=b^{\alpha} \frac{H_{i}}{N_{h}} M_{s}-\gamma^{\alpha} M_{e}-\delta^{\alpha} M_{e} \\
{ }^{C} D^{\alpha} M_{i}=\gamma^{\alpha} M_{e}+\eta^{\alpha} E_{i}-\delta^{\alpha} M_{i} \\
{ }^{C} D^{\alpha} H_{s}=R_{h b}^{\alpha} N_{h}-b^{\alpha} \frac{H_{s}}{N_{h}} M_{i}-R_{h d}^{\alpha} H_{s} \\
{ }^{C} D^{\alpha} H_{i}=b^{\alpha} \frac{H_{s}}{N_{h}} M_{i}-\xi^{\alpha} H_{i}-R_{h d}^{\alpha} H_{i}, \\
{ }^{C} D^{\alpha} H_{r}=\xi^{\alpha} H_{i}-R_{h d}^{\alpha} H_{r} .
\end{array}\right.
$$

The initial values are $H_{s}(0)=341094$ (total human population), $H_{i}(0)=26$ (number of confirmed cases), and $H_{r}(0)=0$ according to the collected info in December, 2010 in FongShan district (Kaohsiung, Taiwan). The transmissible biting rate was taken as 0.33 on a daily basis (meaning that one bite occurs per three days for one female mosquito). At an initial time $t=0$, the values for pre-adult and adult vector populations are set as $E_{s}(0)=0, E_{i}(0)=0, M_{s}(0)=341120$, $M_{e}(0)=0$, and $M_{i}(0)=0$, respectively. The total adult mosquito population $N_{m}$ was 341120 female mosquitoes that is same value with the human population size. The detailed assumptions of the considered (vertical) transmission dengue dynamics model can be seen in [20] and the references therein.

\subsection{Equilibrium Points}

The system has two types of disease free equilibrium points namely trivial disease-free equilibrium (DFE) $E_{0}^{\alpha}$ and biologically realistic diseasefree equilibrium (BRDFE) $\widehat{E}_{1}^{\alpha}$. To find equilibrium points, we will solve the following system:

${ }^{C} D^{\alpha} E_{s}=0,{ }^{C} D^{\alpha} E_{i}=0,{ }^{C} D^{\alpha} M_{s}=0,{ }^{C} D^{\alpha} M_{e}=0$, ${ }^{C} D^{\alpha} M_{i}=0,{ }^{C} D^{\alpha} H_{s}=0,{ }^{C} D^{\alpha} H_{i}=0,{ }^{C} D^{\alpha} H_{r}=0$.

By solving above system, we obtain the following equilibrium points:

$$
\begin{gathered}
E_{0}^{\alpha}=\left(0,0,0,0,0, \bar{H}_{s}, 0,0\right) \\
\widehat{E}_{1}^{\alpha}=\left(\widehat{E}_{s}, 0, \widehat{M}_{s}, 0,0, \widehat{H}_{s}, 0,0\right)
\end{gathered}
$$

where

$$
\bar{H}_{s}=\frac{R_{h b}^{\alpha} N_{h}}{R_{h d}^{\alpha}}, \widehat{E}_{s}=\frac{e_{v}^{\alpha}}{q^{\alpha}}, \widehat{M}_{s}=\frac{e_{v}^{\alpha}}{\delta^{\alpha}}, \widehat{H}_{s}=\frac{R_{h b}^{\alpha} N_{h}}{R_{h d}^{\alpha}} .
$$

\subsection{Stability analysis}

Theorem 1. The biologically realistic diseasefree equilibrium (BRDFE) $\widehat{E}_{1}^{\alpha}$ of the system in Eq. (3) is locally asymptotically stable if

$$
R_{1}^{\alpha}=\frac{N_{m}+\eta^{\alpha} e_{v}^{\alpha}-\eta^{\alpha} \delta^{\alpha} N_{m}}{N_{m}}<1 .
$$

Proof. The Jacobian matrix of the system in Eq. (3) evaluated biologically realistic diseasefree equilibrium (BRDFE) $\widehat{E}_{1}^{\alpha}$ is given by 
Table 1. Dengue model parameters (at temperature $25^{\circ} \mathrm{C}$ )

\begin{tabular}{|c|c|c|c|}
\hline Symbol & Meaning and unit & Range of values & References \\
\hline \hline$p$ & "proportion of eggs" & 0.028 & 33 \\
$\eta$ & "pre-adult mosquito maturation rate (per day)" & 0.099 & Estimated \\
$b$ & "the biting rate (per day)" & 0.33 & 32 \\
$e_{v}$ & "Oviposition rate (per day)" & 6.218 & Estimated \\
$\gamma$ & "Virus incubation rate in mosquito (per day)" & 0.0607 & Estimated \\
$\delta$ & "Adult mosquito death rate (per day)" & 0.0331 & Estimated \\
$\xi$ & "Human recovery rate (per day)" & $1 / 7$ & 32 \\
$R_{h d}$ & "Human death rate (per day)" & 0.000016 & 31 \\
$R_{h b}$ & "Human birth rate(per day)" & 0.00002 & 31 \\
$N_{m}$ & "Total number of mosquitoes" & 341120 & Assumed \\
$N_{h}$ & "Total size of human population" & 341120 & 31 \\
\hline
\end{tabular}

$$
\left[\begin{array}{ccc}
\eta^{\alpha} & 0 & 0 \\
0 & -\eta^{\alpha} & 0 \\
\eta^{\alpha} & 0 & -\delta^{\alpha} \\
0 & 0 & 0 \\
0 & \eta^{\alpha} & 0 \\
0 & 0 & 0 \\
0 & 0 & 0 \\
0 & 0 & 0
\end{array}\right.
$$$$
J\left(\widehat{E}_{1}^{\alpha}\right)=
$$

The calculated eigenvalues are given by

$$
\begin{gathered}
\lambda_{1}=-\eta^{\alpha}, \lambda_{2}=-\delta^{\alpha}, \lambda_{3}=-R_{h d}^{\alpha}, \\
\lambda_{4}=-R_{h d}^{\alpha}, \lambda_{5}=-\left(\xi^{\alpha}+R_{h d}^{\alpha}\right), \lambda_{6}=-\left(\gamma^{\alpha}+\delta^{\alpha}\right),
\end{gathered}
$$

remaining eigenvalues are the roots of the quadratic polynomial

$$
\lambda^{2}+\left(\eta^{\alpha}+\delta^{\alpha}\right) \lambda+\left(1-R_{1}^{\alpha}\right)=0,
$$

by the Routh-Hurwitz stability criterion, $\widehat{E}_{1}^{\alpha}$ is locally asymptotically stable if and only if $R_{1}^{\alpha}<1$.

\subsection{The basic reproduction number $\left(R_{0}^{\alpha}\right)$}

The basic reproduction number $R_{0}^{\alpha}$ of the epidemic disease is known as the number of secondary infections caused by a unique infected individual. Hypothetically, when $R_{0}^{\alpha}<1$ the transmissions chains are not beneficial and disease will eradicate from the population. Although if $R_{0}^{\alpha}>1$, each generation will be raised by the number of infected humans and infection will be taken advantage of with the usage of next generation matrix approach. In this situation $R_{0}^{\alpha}$ is equal to the spectral radius of $K=F V^{-1}$, where $F$ is a non-negative matrix of the infection items, and $V$ is the $M$-Matrix of the corresponding transition terms given as in below 13

$$
\begin{aligned}
& \left.\begin{array}{l}
0 \\
0 \\
0 \\
0 \\
0
\end{array}\right] \quad F=\left(\begin{array}{cccc}
0 & 0 & 0 & b^{\alpha} \\
0 & 0 & 0 & p \delta \\
b^{\alpha} \frac{N_{m}}{N_{h}} & 0 & 0 & 0 \\
0 & 0 & 0 & 0
\end{array}\right) \text {, } \\
& \left.\begin{array}{c}
0 \\
-R_{h d}^{\alpha}
\end{array}\right] \quad V^{-1}=\left(\begin{array}{cccc}
\frac{1}{\xi^{\alpha}+R_{h d}^{\alpha}} & 0 & 0 & 0 \\
0 & \frac{1}{\eta^{\alpha}} & 0 & 0 \\
0 & 0 & \frac{1}{\gamma^{\alpha}+\delta^{\alpha}} & 0 \\
0 & \frac{1}{\eta^{\alpha}} & \frac{1}{\gamma^{\alpha}} & \frac{1}{\delta^{\alpha}}
\end{array}\right) \text {, }
\end{aligned}
$$

and so

$$
F V^{-1}=\left(\begin{array}{cccc}
0 & \frac{b^{\alpha}}{\delta^{\alpha}} & \frac{b^{\alpha}}{\gamma^{\alpha}} & \frac{b^{\alpha}}{\delta^{\alpha}} \\
0 & p & \frac{p \delta^{\alpha}}{\gamma^{\alpha}} & p \\
\frac{b^{\alpha} N_{m}}{N_{h}}\left(\xi^{\alpha}+R_{h d}^{\alpha}\right) & 0 & 0 & 0 \\
0 & 0 & 0 & 0
\end{array}\right) .
$$

The eigenvalues of $F V^{-1}$ is:

$$
\frac{p}{2} \pm \frac{1}{2} \sqrt{p^{2}+\frac{4 b^{\alpha} N_{m}}{N_{h} \gamma^{\alpha}\left(\xi^{\alpha}+R_{h d}^{\alpha}\right)}}
$$

Therefore, the dominant eigenvalue of $F V^{-1}$ is

$$
R_{0}^{\alpha}=\frac{p}{2}+\frac{1}{2} \sqrt{p^{2}+\frac{4 b^{2 \alpha} N_{m}}{N_{h} \gamma^{\alpha}\left(\xi^{\alpha}+R_{h d}^{\alpha}\right)}} .
$$

\section{Numerical method and simulations}

In this section, we will construct the discretization of the model given by Eq. (3) involving the Caputo fractional operator.

First, we rewrite system in Eq. (3) in a compact form so that the relations can be simplified

$$
\left\{\begin{array}{l}
{ }^{C} D^{\alpha} Y(t)=\mathcal{F}(Y(t)), \quad 0<t<b<\infty \\
Y(0)=Y^{0}
\end{array}\right.
$$

where $Y=\left(E_{s}, E_{i}, M_{s}, M_{e}, M_{i}, H_{s}, H_{i}, H_{r}\right) \in$ $R_{+}^{8}$, where $\mathcal{F}$, is a real-valued continuous vector 
function having the Lipschitz condition $\left\|\mathcal{F}\left(Y_{1}(t)\right)-\mathcal{F}\left(Y_{2}(t)\right)\right\| \leq L\left\|Y_{1}(t)-Y_{2}(t)\right\|, \quad L>0$,

and $Y^{0}=\left(E_{s}^{0}, E_{i}^{0}, M_{s}^{0}, M_{e}^{0}, M_{i}^{0}, H_{s}^{0}, H_{i}^{0}, H_{r}^{0}\right)$ is the initial vector. By applying the fractional integral operator in Eq. (2) to Eq. (6) the following result is obtained

$$
Y(t)=Y_{0}+{ }^{C} I^{\alpha} \mathcal{F}(Y(t)), \quad 0<t<b<\infty,
$$

where ${ }^{C} I^{\alpha}$ represents the Riemann-Liouville integral operator. In order to propose a numerical scheme, the discretization of the time interval $[0, b]$ is done by equally spaced nodes so that time step size is $\tau=\frac{b}{N}$. Let $Y_{n}$ denote the approximation of the true solution $Y\left(t_{n}\right)$ at point $t_{n}=n \tau$ and $0=t_{0}<t_{1}<\ldots<t_{N}=b$, with $t_{n+1}-t_{n}=$ $\tau, n=0,1,2, \ldots,(N-1)$. Then, we derive following numerical scheme for the Caputo operator using Euler method 34

$$
Y^{n+1}=Y^{0}+\frac{\tau^{q}}{\Gamma(\alpha+1)} \sum_{k=0}^{n} w_{k+1, j} \mathcal{F}\left(Y_{k}\right),
$$

where $(\mathrm{n}=0,1,2, \ldots,(\mathrm{N}-1))$ and

$$
w_{k+1, j}=(k+1-j)^{\alpha}-(k-j)^{\alpha}
$$

are the weights of the fractional Euler method. Thus, we obtain the following discretization of the model in Eq. (3)

$$
\left\{\begin{array}{l}
E_{s}^{n+1}=E_{s}^{0} \\
+\frac{\tau^{q}}{\Gamma(\alpha+1)} \sum_{k=0}^{n} w_{k+1, j}\left(e_{v}^{\alpha}\left(1-p\left(\frac{M_{i}^{k}}{N_{m}}\right)\right)-\eta^{\alpha} E_{s}^{k}\right) \\
E_{i}^{n+1}=E_{i}^{0} \\
+\frac{\tau^{q}}{\Gamma(\alpha+1)} \sum_{k=0}^{n} w_{k+1, j}\left(e_{v}^{\alpha} p\left(\frac{M_{i}^{k}}{N_{m}}\right)-\eta^{\alpha} E_{i}^{k}\right) \\
M_{s}^{n+1}=M_{s}^{0} \\
+\frac{\tau^{q}}{\Gamma(\alpha+1)} \sum_{k=0}^{n} w_{k+1, j}\left(\eta^{\alpha} E_{s}^{k}-b^{\alpha} \frac{H_{i}^{k}}{N_{h}} M_{s}^{k}-\delta^{\alpha} M_{s}^{k}\right) \\
M_{e}^{n+1}=M_{e}^{0} \\
+\frac{\tau^{q}}{\Gamma(\alpha+1)} \sum_{k=0}^{n} w_{k+1, j}\left(b^{\alpha} \frac{H_{i}^{k}}{N_{h}} M_{s}^{k}-\gamma^{\alpha} M_{e}^{k}-\delta^{\alpha} M_{e}^{k}\right) \\
M_{i}^{n+1}=M_{i}^{0} \\
+\frac{\tau^{q}}{\Gamma(\alpha+1)} \sum_{k=0}^{n} w_{k+1, j}\left(\gamma^{\alpha} M_{e}^{k}+\eta^{\alpha} E_{i}^{k}-\delta^{\alpha} M_{i}^{k}\right), \\
H_{s}^{n+1}=H_{s}^{0} \\
+\frac{\tau^{q}}{\Gamma(\alpha+1)} \sum_{k=0}^{n} w_{k+1, j}\left(R_{h b}^{\alpha} N_{h}-b^{\alpha} \frac{H_{s}^{k}}{N_{h}} M_{i}^{k}-R_{h d}^{\alpha} H_{s}^{k}\right), \\
H_{i}^{n+1}=H_{i}^{0} \\
+\frac{\tau^{q}}{\Gamma(\alpha+1)} \sum_{k=0}^{n} w_{k+1, j}\left(b^{\alpha} \frac{H_{s}^{k}}{N_{h}} M_{i}^{k}-\xi^{\alpha} H_{i}^{k}-R_{h d}^{\alpha} H_{i}^{k}\right), \\
H_{r}^{n+1}=H_{r}^{0} \\
+\frac{\tau^{q}}{\Gamma(\alpha+1)} \sum_{k=0}^{n} w_{k+1, j}\left(\xi^{\alpha} H_{i}^{k}-R_{h d}^{\alpha} H_{r}^{k}\right),
\end{array}\right.
$$

where $n=0,1,2, \ldots,(N-1)$, and $N_{m}=M_{s}+$ $M_{e}+M_{i}$.

\subsection{Simulation results and discussion}

We solve the newly proposed fractional dengue model given by Eq. (3) numerically. The effect of temperature on the dynamics of dengue epidemics is presented by Fig 2 where the temperature values are considered as $18^{\circ} \mathrm{C}, 25^{\circ} \mathrm{C}$ and $28^{\circ} \mathrm{C}$ since the parameter values in Table 1 are known at these temperatures. It is stated that (see ref. [20] and the related references therein) $e_{v}$-oviposition rate, $\gamma$-virus incubation rate in mosquito, $\delta$-adult mosquito death rate and $\eta$-pre-adult mosquito maturation rate are found as temperature dependent entomological parameters in the selected district of Taiwan. It is seen that the temperature is a significant environmental factor that affect the transmission in dengue epidemics. The obtained results in Fig 2 shows that the infected human (host) population and infected (pre-adult and adult) mosquito populations reached to their peak values with a higher amount at the temperature of $28^{\circ} \mathrm{C}$ approximately in the period of 50-100 days. Correspondingly, the susceptible human population showed a sharper decrease whereas the recovered human population size has a sharper increase (after 100 days approx.) at $28^{\circ}$ C.

The impact of the fractional order is depicted in Fig 3. In this sense, the temperature effect in vector-host (vertical) transmission dynamics in dengue epidemics formulated by fractional order dengue model is investigated for different values of the fractional order such as $\alpha=1,0.8$ and $\alpha=0.6$ at a fixed temperature of $25^{\circ} \mathrm{C}$. The results for all considered values of $\alpha$ reach to steady state for each population and integer order case is recovered when $\alpha=1$. The susceptible human and pre-adult mosquito populations, recovered human populations reach to different values in the asymptotic case as $\alpha$ differs for the fixed temperature value of $25^{\circ} \mathrm{C}$. The fractional order $\alpha$ here enables us to include the memory into the evolution of the epidemic dynamics and also it gives us the opportunity to capture different behaviors of the system components inside the same model.

Moreover, Table 2 verify the Theorem 1 as the ,condition $R_{1}^{\alpha}<1$ is satisfied for the considered 3 -different temperature cases which means that the biologically realistic disease free equilibriums of the system are all asymptotically stable.

Table 2. Stability of $\widehat{E}_{1}^{\alpha}$

\begin{tabular}{cccc}
\hline Equilibrium & $R_{1}^{1}$ & $R_{1}^{0.8}$ & $R_{1}^{0.6}$ \\
\hline \hline$\widehat{E}_{1}^{\alpha}$ at $18^{\circ} \mathrm{C}$ & 0.9761 & 0.9931 & 0.9761 \\
$\widehat{E}_{1}^{\alpha}$ at $25^{\circ} \mathrm{C}$ & 0.9677 & 0.9897 & 0.9677 \\
$\widehat{E}_{1}^{\alpha}$ at $28^{\circ} \mathrm{C}$ & 0.9633 & 0.9878 & 0.9633 \\
\hline
\end{tabular}




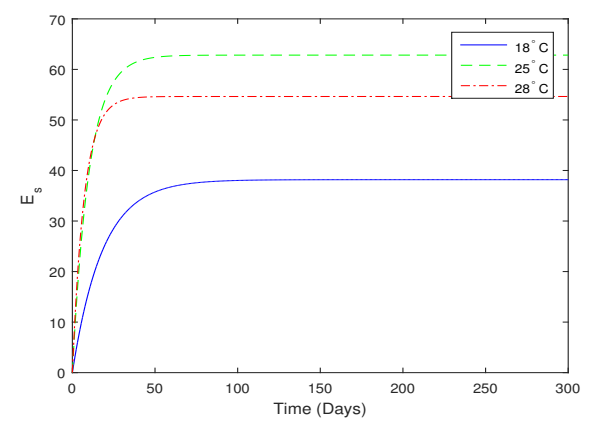

(a) Pre-adult female mosquito-Susceptible

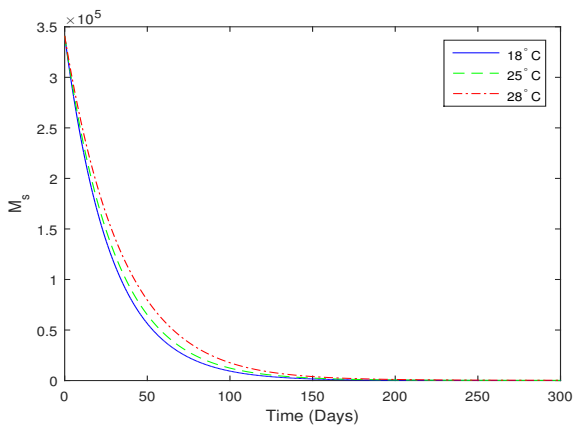

(c) Adult female mosquito-Susceptible

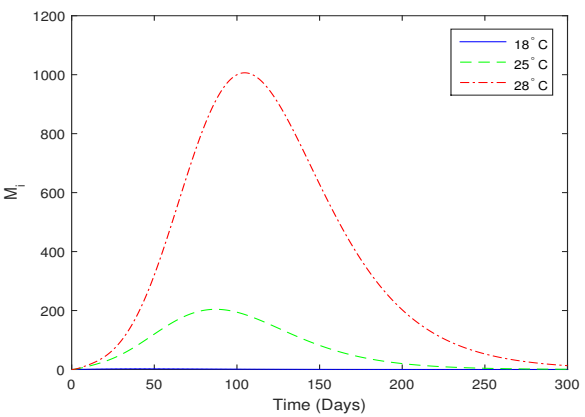

(e) Adult female mosquito-Infectious

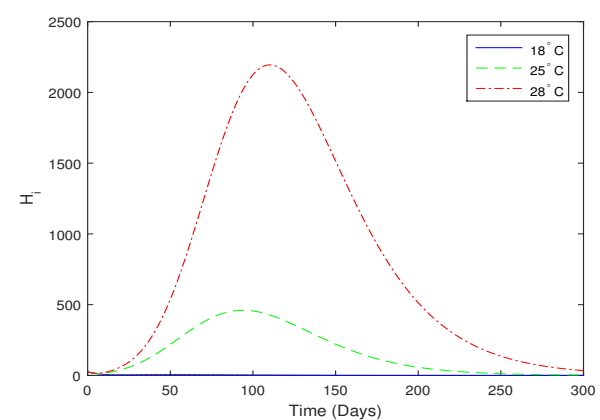

(g) Human population-Infected/Infectious

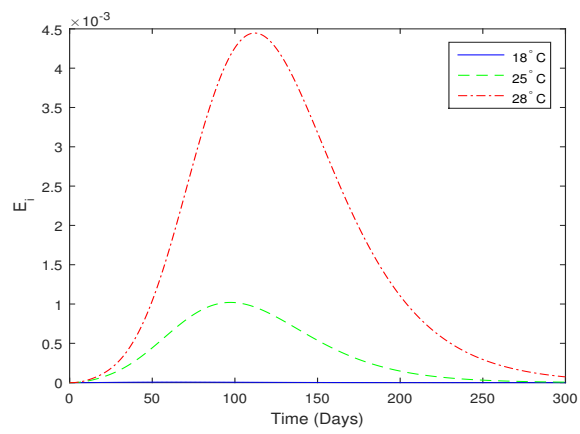

(b) Pre-adult female mosquito-Infected

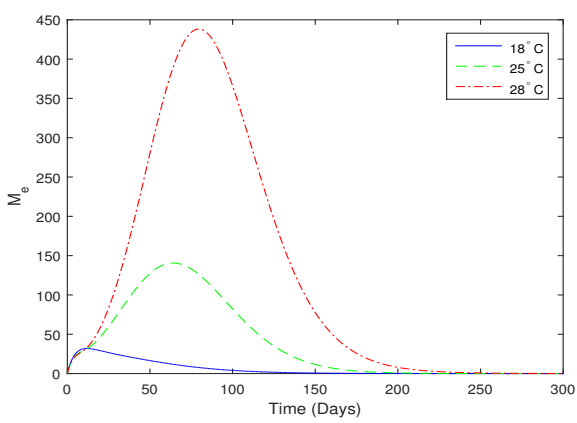

(d) Adult female mosquito-Infected

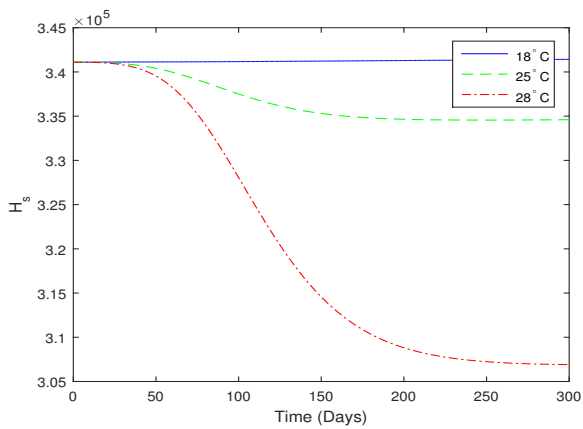

(f) Human population-Susceptible

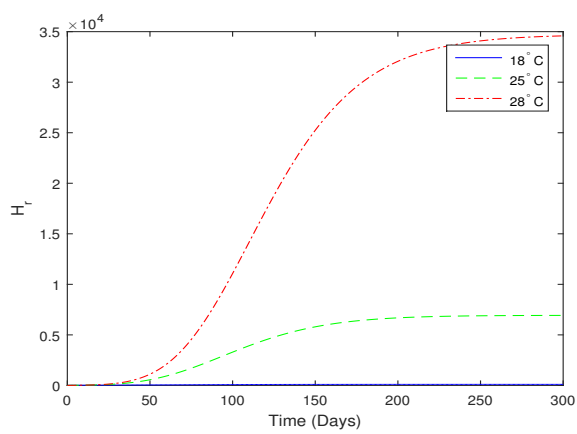

(h) Human population-Recovered/Immune

Figure 2. Simulation results of the fractional order dengue model at temperature $18^{\circ} \mathrm{C}, 25^{\circ}$ $\mathrm{C}$ and $28^{\circ} \mathrm{C}$ with $\alpha=1$.

\section{Conclusion}

A fractional order dengue model is newly investigated that incorporate temperature dependent features in entomological parameters. In the modeling process four entomological parameters are used for this purpose, that includes the oviposition rate $\left(e_{v}\right)$, pre-adult mosquito maturing rate $\eta$, adult mosquito death rate $\delta$, and the virus incubation rate in mosquitoes $\gamma$. Weekly mean temperature data in Kaohsiung (2001-2010) 20] has been utilized to perform the simulations. We applied $L 1$ method to solve the model numerically for various fractional order and temperatures. Further, the basic reproduction number is derived for the newly considered fractional order 


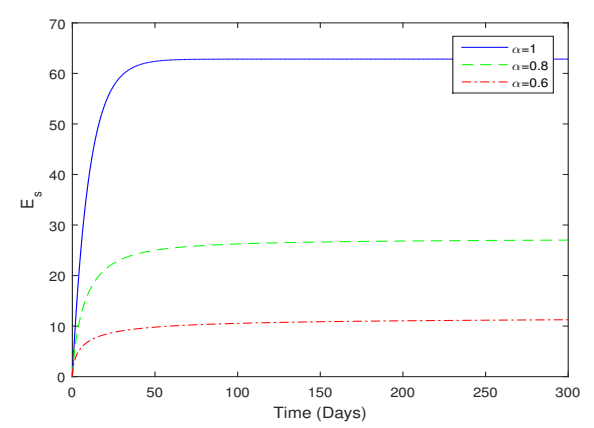

(a) Pre-adult female mosquito-Susceptible

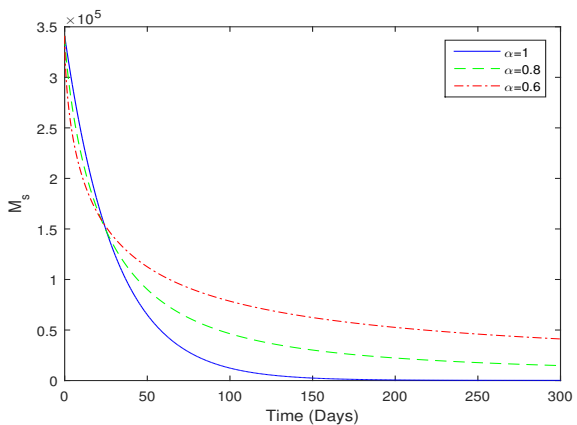

(c) Adult female mosquito-Susceptible

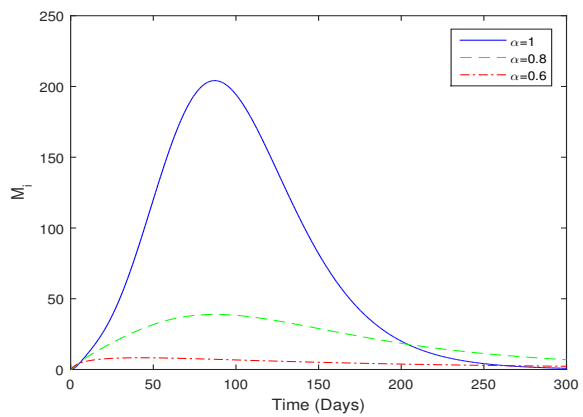

(e) Adult female mosquito-Infectious

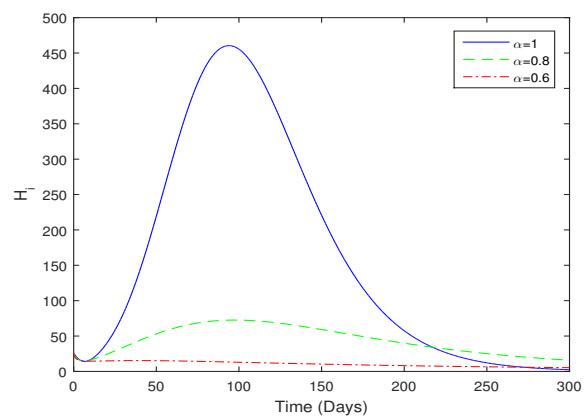

(g) Human population-Infected/Infectious

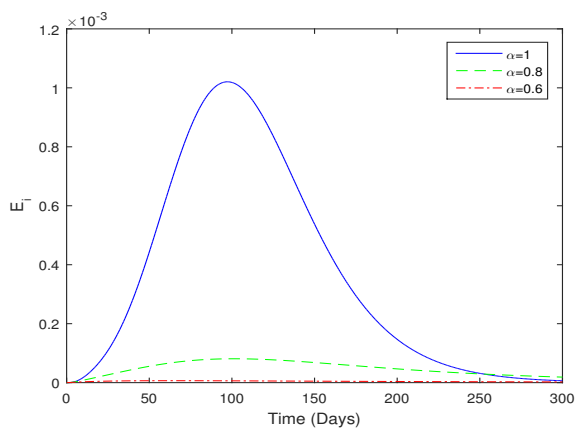

(b) Pre-adult female mosquito-Infected

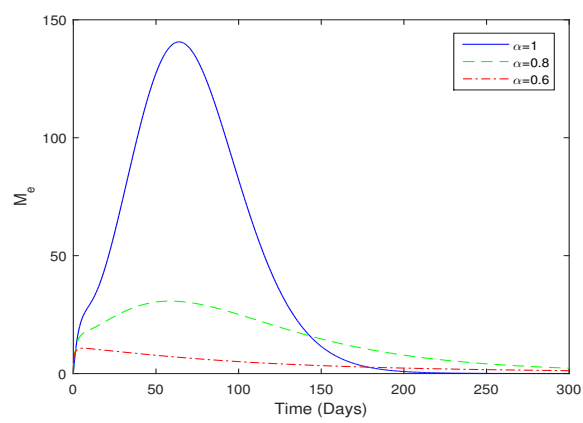

(d) Adult female mosquito-Infected

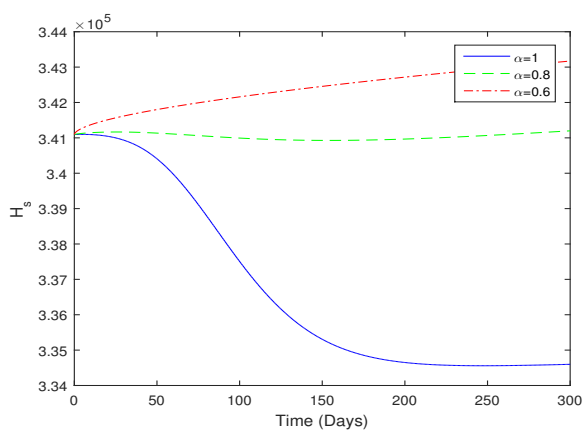

(f) Human population-Susceptible

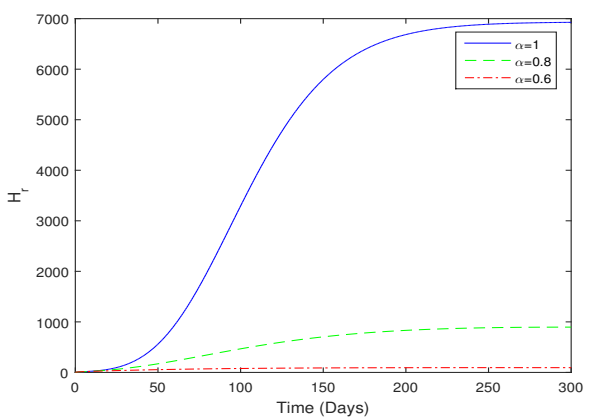

(h) Human population-Recovered/Immune

Figure 3. Simulation results of the fractional order dengue model at $\alpha=1, \alpha=0.8, \alpha=0.6$ at temperature $25^{\circ} \mathrm{C}$.

dengue model (that is formulated by using Caputo fractional operators). Correspondingly, stability analysis is performed and the local asymptotic stability of the disease-free equilibria is obtained. Simulation results refers that the highest danger of dengue transmission exists at temperature $28^{\circ}$ C. The control of dengue transmission is based on the control of the aquatic and adult stages of the mosquito and so this study can contribute to adapt effective control strategies like the use of pesticide and other techniques. 


\section{Acknowledgments}

This research is supported by the Scientific and Technological Research Council of Turkey (TUBITAK) under the Grant No. TBAG117F473 and also the COST Actions CA16227, CA15225 as networks supported by COST (European Cooperation in Science and Technology).

\section{References}

[1] World Health Organization(WHO). (2019). Dengue Situation Update [online]. Available from: https:www.who. int/westernpacific/emergencies/ surveillance/dengue [Accessed August 2019]

[2] World Health Organization(WHO). (2019). Dengue and Severe Dengue [online]. Available from: https:www.who. int/news-room/fact-sheets/detail/ dengue-and-severe-dengue [Accessed June 2019]

[3] United States Environmental Protection Agency(EPA). (2019). Mosquito Life Cycle [online]. Available from: https:www.epa.gov/mosquitocontrol/ mosquito-life-cycle [Accessed July 2019]

[4] Chowell, G., Diaz-Duenas, P., Miller, J.C., Alcazervelazco, A., Hyman, J.M., Fenimore, P.W., \& Chavez, C. (2007). Estimation of the reproduction number of dengue fever from spatial epidemic data. Mathematical Biosciences, 208, 571-589.

[5] Derouich, M., \& Boutayeb, A. (2006). Dengue fever: Mathematical modelling and computer simulation. Applied Mathematics and Computation, 177, 528-544.

[6] Dietz, K. (1974). Transmission and control of arbo virus diseases. SIAM, 975, 104-21.

[7] Esteva, L., \& Vargas, C. (1998). Analysis of dengue transmission model. Mathematical Biosciences, 150(2), 131-51.

[8] Estweva, L., \& Yang, H. (2005). Mathematical model to assess the control of Aedes aegypti mosquitoes by the sterile insect technique. Mathematical Biosciences, 198(2), 132-47.

[9] Garba, S.M., Gumel, A.B., \& Abu Bakar, M.R. (2008). Backward bifurcations in dengue transmission dynamics. Mathematical Biosciences, 215(1), 11-25.

[10] Thome, R.C., Yang, H.M., \& Esteva, L. (2010). Optimal control of Aedes aegypti mosquitoes by the sterile insect technique and insecticide. Mathematical Biosciences, 223(1), $12-23$.
[11] Pinho, S.T.R., et al. (2010). Modelling the dynamics of dengue real epidemics. Philosophical Transactions of The Royal Society, 368, 5679-5693.

[12] Sardar, T., Rana, S., \& Chattopadhyay, J. (2015). A mathematical model of dengue transmission with memory. Communnications in Nonlinear Science and Numerical Simulation, 22(1), 511-525.

[13] Stanislavsky, A. (2000). Memory effects and macroscopic manifestation of randomness. Physical Review E, 61(5), 4752-4759.

[14] Syafruddin, S., \& Noorani, M.S.M. (2012). SEIR model for transmission of dengue fever in Selangor Malaysia. International Journal of Modern Physics: Conference Series, 9, 380389.

[15] Abdelrazec, A., Belair, J., Shan, C., \& Zhu, Y. (2016). Modeling the spread and control of dengue with limited public health resources. Mathematical Biosciences, 271, 136-145.

[16] Andraud, M., Hens, N., Marais, C., \& Beutels, P. (2012). Dynamic epidemiological models for dengue transmission: a systematic review of structural approaches. PloS One, $7(11)$, e49085.

[17] Robert, Michael A., Christofferson, R.C., Weber, P.D., \& Wearing, H.J. (2019). Temperature impacts on dengue emergence in the United States: Investigating the role of seasonality and climate change. Epidemics, 28, UNSP 10034.

[18] Alto, B., \& Bettinardi, D. (2013). Temperature and dengue virus infection in mosquitoes: Independent effects on the immature and adult stages. The American Journal of Tropical Medicine and Hygiene, 88, 497-505.

[19] Taghikhani, R., \& Gumel, A.B. (2018). Mathematics of dengue transmission dynamics: Roles of vector vertical transmission and temperature fluctuations. Infectious Disease Modelling, 3, 266-292.

[20] Chen, S.C., \& Hsieh, M.H. (2012). Modeling the transmission dynamics of dengue fever: Implications of temperature effects. Science of the Total Environment, 431, 385-391.

[21] Yang, H.M., Macoris, M.L.G., Galvani, K.C., Andrighetti, M.T.M., \& Wanderley, D.M.V. (2009). Assessing the effects of temperature on dengue transmission. Epidemiology $\mathcal{E}$ Infection, 137(8), 1179-1187.

[22] Hamdan, N.I., \& Kilicman, A. (2018). A fractional order SIR epidemic model for dengue transmission. Chaos, Solutions and Fractals, $114,55-62$. 
[23] Ozalp, N., \& Demirci E. (2011). A fractional order SEIR model with vertical transmission. Mathematical and Computer Modelling, 54(12), 1-6.

[24] Qureshi, S., Yusuf, A., Shaikh, A.A., \& Inc, M. (2019). Transmission dynamics of varicella zoster virus modeled by classical and novel fractional operators using real statistical data. Physica A: Statistical Mechanics and its Applications, 534, 122149.

[25] Qureshi, S., \& Yusuf, A. (2019). Fractional derivatives applied to MSEIR problems: Comparative study with real world data. The European Physical Journal Plus, 134 (4), 171 (13 pages).

[26] Pooseh, S., Rodrigues, H.S., \& Torres, D.F.M. (2011). Fractional derivatives in dengue epidemics. In: T.E. Simos, G. Psihoyios, C. Tsitouras and Z. Anastassi, eds. Numerical Analysis and Applied Mathematics, American Institute of Physics, Melville, 739-742.

[27] Diethelm, K. (2013). A fractional calculus based model for the simulation of an outbreak of dengue fever. Nonlinear Dynamics, 71, 613619.

[28] Qureshi, S., \& Atangana, A. (2019). Mathematical analysis of dengue fever outbreak by novel fractional operators with field data. Physica A, 526, 121127.

[29] Jajarmi, A., Arshad, S., \& Baleanu, D. (2019). A new fractional modelling and control strategy for the outbreak of dengue fever. Physica A: Statistical Mechanics and its Applications, Volume 535, 122524.

[30] Samko, S.G., Kilbas, A.A., \& Marichev, O.I. (1993). Fractional Integrals and Derivatives:
Theory and Applications. Gordon and Breach, New York.

[31] Civil Affairs Bureau, Kaohsiung city government. Available from [online]: http: cabu.kcg.gov.tw/cabu2/statis61B2.aspx [Accessed December 2011]

[32] Adams, B., \& Boots, M. (2010). How important is vertical transmission in mosquitoes for the persistence of dengue? Insights from a mathematical model. Epidemics, 2, 1-10.

[33] Joshi, V., Mourya, D.T., \& Sharma, R.C. (2002). Persistence of dengue-3 virus through transovarial transmission passage in successive generations of Aedes aegypti mosquitoes. American Journal of Tropical Medicine and Hygiene, 67(2), 158-61.

[34] Li, C., \& Zeng, F. (2013). The finite difference methods for fractional ordinary differential equations. Numerical Functional Analysis and Optimization, 34(2), 149-79.

Ozlem Defterli received her B.Sc. degree major in Mathematics and Computer Science and minor in Computer Engineering from Cankaya University (Ankara, Turkey) with full scholarship. After completing her M.Sc. education in Mathematics and Computer Science Program (with full scholarship) in the same university, she got her Ph.D. degree from the Department of Mathematics in Middle East Technical University (METU) in August 2011. She has been a faculty member in Cankaya University - Department of Mathematics since 2002. Her research interests lie in the areas of numerical methods for classical and fractional order differential equations, applied optimal control, mathematical modelling and mathematical biology.

(10) http://orcid.org/0000-0003-0918-9834

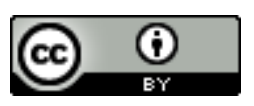

This work is licensed under a Creative Commons Attribution 4.0 International License. The authors retain ownership of the copyright for their article, but they allow anyone to download, reuse, reprint, modify, distribute, and/or copy articles in IJOCTA, so long as the original authors and source are credited. To see the complete license contents, please visit http://creativecommons.org/licenses/by/4.0/. 